\title{
Giant sialolith at sublingual salivary gland. Biggest?
}

\author{
Abul Fauzi, Irfan Rasul
}

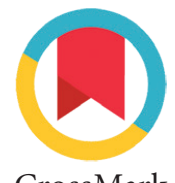

Abstract

Objective: Sialoliths or salivary gland duct calculus are the most common pathologies of the salivary gland. The majority of sialoliths occur in the submandibular gland or its duct and are a common cause of acute and chronic infections. Sialoliths are deposits obstructing the ducts of major or minor salivary glands.

Methods: Clinical and radiographic examination panoramic and CBCT was conducted and the diagnosis is sialolithiasis on salivary ducts sublingual gland sinistra and removal of salivary glands stone (sialolithotomy) was planned intraorally with sialodochoplasty under general anesthesia.

Results: CBCT and intra operation obtained the stone in the wharton's duct with $26.5 \mathrm{~mm}$ in length and $17 \mathrm{~mm}$ in diameter.

Conclusion: The main complaint of sialolithiasis is pain accompanied by swelling. The therapy that can be done for this sialolith is divided into conventional techniques without surgery, surgical therapy and therapy with Extracorporeal Shock Wave Lithotripter (ECSWL) techniques.
Department of Oral and Maxilllofacial Surgery, Faculty of Dentistry, Hasanuddin University, Makassar, Indonesia
${ }^{*}$ Corresponding to:

Abul Fauzi, Department of Oral and Maxilllofacial Surgery, Faculty of Dentistry, Hasanuddin University, Makassar, Indonesia abulfauzi@gmail.com
Received: 16 January 2018 Revised: 10 June 2018 Accepted: 9 July 2018 Available Online 1 August 2018

Keywords: Giant sialolith, Sublingual salivary gland

Cite this Article: Fauzi A, Rasul I. 2018. Giant sialolith at sublingual salivary gland. Biggest?. Journal of Dentomaxillofacial Science 3(2): 123-125. DOI: 10.15562/jdmfs.v3i2.704

\section{Introduction}

The stone blockage or calcification that develops in the salivary glands or ducts is known as sialolithiasis. The pain caused by this blockage is very disturbing to the patients, especially during a meal and slowly decreases after eating. These symptoms will continue to appear when the salivary glands are stimulated to secrete saliva, such as during a meal.

Sialolithiasis is a common salivary disease in approximately $65 \%$ of patients with chronic sialadenitis. The incidence of sialolith is $80 \%$ in the submandibular gland, $19 \%$ in the parotid gland and $1 \%$ in the sublingual gland. The occurrence of sialolithiasis is twice as high in men with peak incidence at age between 30 and 50 years. ${ }^{1}$

Sialolithiasis is an infection caused by a stone or calcified structure that develops in the salivary glands or the ducts, which can occur in the major salivary glands and minor salivary glands. Major salivary glands are more commonly affected by sialolithiasis compared with minor salivary glands. Glandular stones may occur in all ductal systems throughout the body such as kidney stones (uroliths) that usually consist of calcium deposits and this condition develops due to hypercalcemia and hypercalciuria and choleliths containing cholesterol, bilirubin which are common in conditions of infection or static state within the bilirubin channel. ${ }^{2}$

\section{Case Report}

A 57 years old man came with a major complaint of pain and swelling on the left side of the base of the mouth that has been occured since the previous 3 months. There was a history of controlled Diabetes mellitus, a sharply penetrating feeling of pain radiating to the tongue causing limited tongue movement. The extraoral examination was within normal limits, while in the intraoral examination, there was reddish edema on the left side of the base of the mouth, with a mild pressure on palpation, there was push out of the duct orifice figure 1 with spongy consistency and hard in some parts.

To find the possibility of tooth infection, a panoramic radiography was conducted to get a radiopaque image figure 2.

In order to determine the size and position of the radiopaque image, Cone Beam Computed Tomography (CBCT) obtained the stone image in

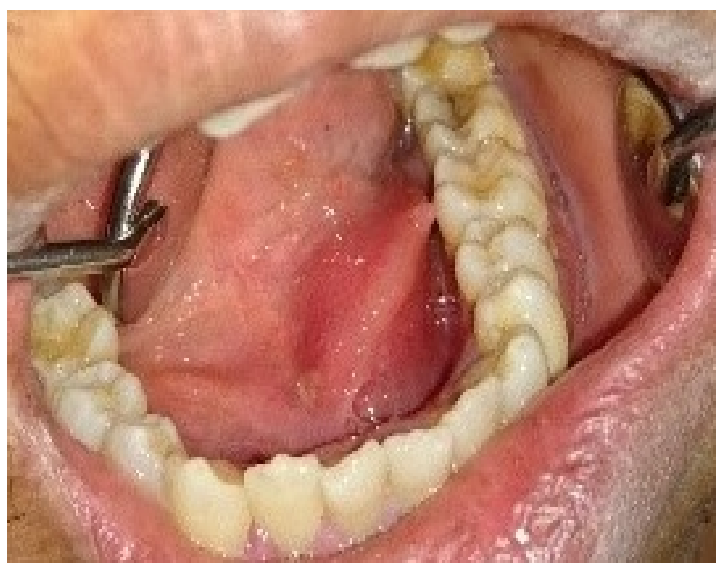

Figure 1 Reddish edema was seen on the left side of the base of the mouth in intraoral picture 


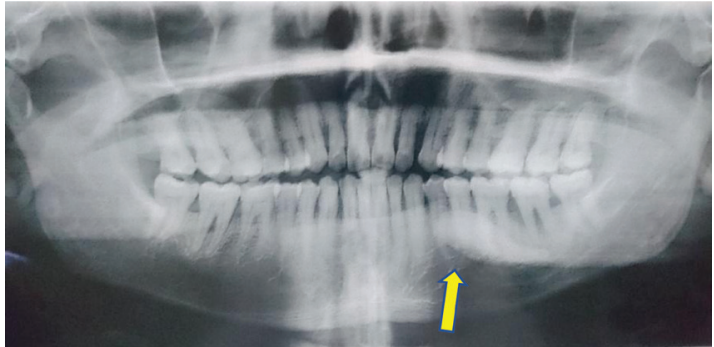

Figure 2 Panoramic image shows radiopaque image of the wharton's duct region

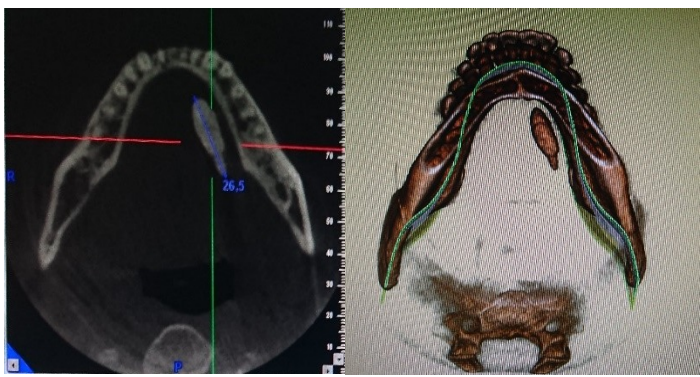

A

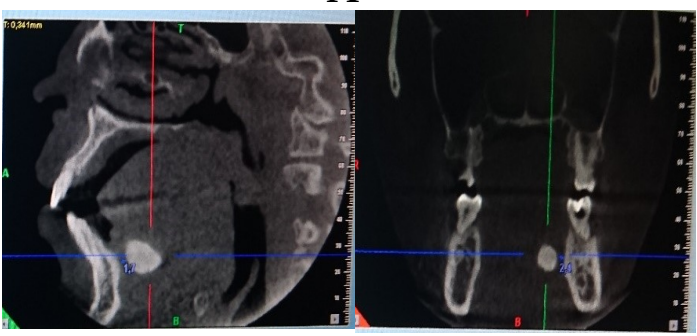

B

Figure 3 A. Sagittal split, B. Transversal split
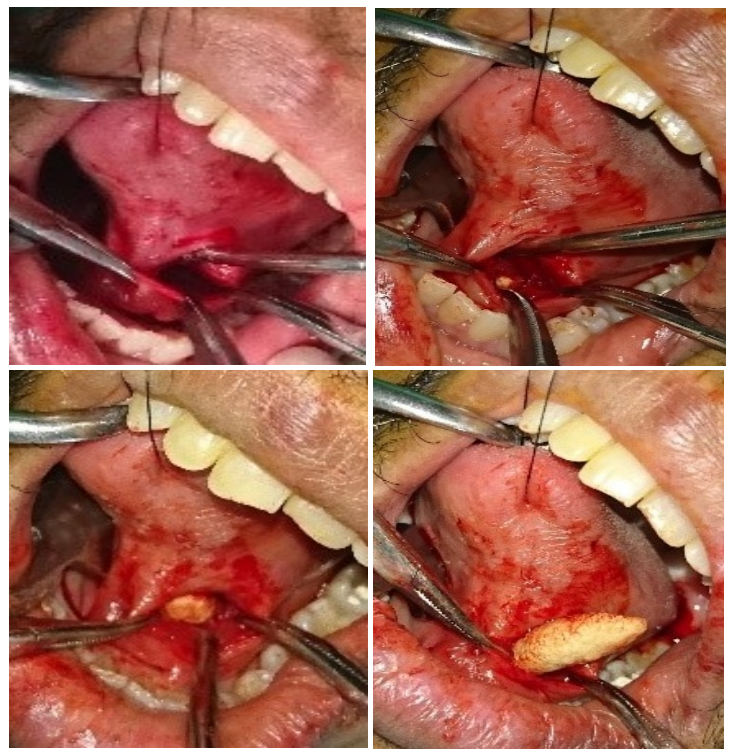

Figure 4 Intraoperative procedure of sialolithotomy at the base of the mouth

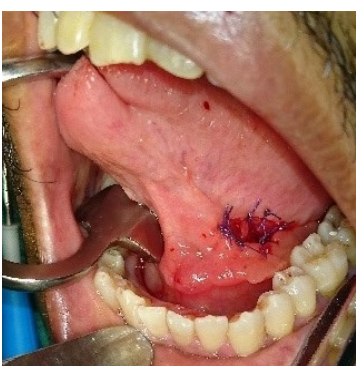

A

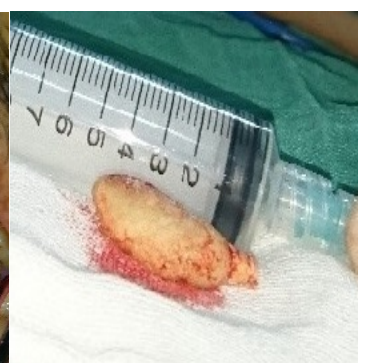

B
Figure 5 A. Post operation sialolithotomy, B. The sialolith with $26.5 \mathrm{~mm}$ in length and $17 \mathrm{~mm}$ in diameter

the Wharton's duct with $26.5 \mathrm{~mm}$ in length and $17 \mathrm{~mm}$ in diameter. Figure 3A shows sagittal split and figure $3 \mathrm{~B}$ shows transversal split.

\section{Methods}

Based on anamnesis, the clinical and radiographic examination established diagnosis of sialolithiasis on salivary ducts sublingual gland sinistra and the removal of salivary glands stone (sialolithotomy) was planned intraorally with sialodochoplasty method and general anesthesia.

Sialolithotomy was performed by making a $2 \mathrm{~cm}$ long incision using blade no. 15 at the base of the mouth longitudinally parallel to the Wharton's duct as you see at figure 4. This was done to avoid sublingual duct injury at the time of tissue opening. The incision was made longitudinally along the ductal mucosa with a blade no. 15. Blunt disinfection was done until the duct is identified and fixation with a suture loop was conducted to facilitate ductal exploration. Once the stone was visible, it was able to be removed easily. Duct wall was sewn on the base mucosa of the mouth to maintain salivary drainage. this surgical action is called sialo dochoplasty (revised action on the salivary gland ducts). To prevent the occurrence of stenosis due to the formation of scar tissue, then a rubber drain was interested into the new orifice for approximately 4 days antibiotics and analgesics were given. After 1 (one) day treatment in hospital, the patient was discharged, the control was conducted on day 3 and 6 post operation figure $5 \mathrm{~A}$ and figure $5 \mathrm{~B}$.

\section{Discussion}

Sialolith is generally 5 to $10 \mathrm{~mm}$ in size and mainly consists of calcium phosphate and small amounts of carbonate in the form of hydroxyapatite. ${ }^{3}$ The 
most common complaint is pain followed by swelling of the salivary glands due to blockage of salivary flow.

Sialolith is from deposition or precipitate of calcium salt around the pre-existing debris nodes, within the ductal lumen. The debris includes the thickening or compaction of the mucus, proteins, and epithelial cells of the desquamated duct or the presence of a foreign object. The intermittent static state can change the mucoid element of saliva into gel, so the gel is a constituent of salt deposition and organic matter into stone. The addition of the layers on the salivary stone continues to increase after calcium salt deposits, to reach even larger size of ductal lumen and lead to blockage of the salivary gland ducts. ${ }^{2,4}$

Diagnosis for salivary glands is fairly easy with clinical examination of extraoral palpation of the salivary gland, bimanual palpation of the duct and observation of quantity and consistency of salivary flow. The confirmation of the diagnosis can be accomplished by investigation such as plain $\mathrm{x}$-ray photographs, ultrasonography, sialography or sialoendoscopy and CT scan.

In the sublingual gland, the examination is performed by bimanual palpation of the third of the anterior base of the mouth. The minor salivary glands are examined by palpation and observation on the surface of the lips, buccal mucosa, palate and the base of the mouth. The blockage of the sublingual gland is very rare, but when this happens it usually clogs on the same side of the oral cavity. Although sialolithiasis rarely occurs in the sublingual saliva glands and minor salivary glands, the necessary action simple excision on its glands. ${ }^{5,6}$

Salivary glands are generally seen with radiopaque and easily identifiable with plain photographs such as panoramic view, photo occlusal, submentovertex view and lateral jaw photo, but there is also a gland stone that has not been fully calcified so it can be seen with radiolucent image and can not be detected by plain photographs alone. Sialography is needed to detect the presence of this type of stone. The therapy that can be done for this sialolith is divided into conventional techniques without surgery, surgical therapy and therapy with Extracorporeal Shock Wave Lithotripter (ECSWL) techniques.

Surgical therapy can be performed if conventional techniques are unsuccessful, this technique can be done by making an incision on the base of mouth so that the duct is wide open and the stone can be removed easily. The stone is attached to the proximal portion of the suture so as not so as not to move to the posterior during surgery. The incision is made longitudinally along the ductal mucosa with a blade no. 15, blunt disinfection is done until the duct is identified and the fixation with loop suture is conducted to facilitate ductal exploration. Once the stone is visible, it can be removed easily. Duct wall is sewn on the base mucosa of the mouth to maintain salivary drainage.

\section{Conclusion}

Sialolithiasis is a term that describes the presence of a stone or calcified structure that develops in the salivary glands or ducts, which can occur in the major salivary glands and minor salivary glands. The main complaint of sialolithiasis is pain accompanied by swelling. The therapy that can be done for this sialolith is divided into conventional techniques without surgery, surgical therapy and therapy with ECSWL techniques.

\section{Aknowledgment}

The authors would like to thank the patient who has been willing to share his case for reported.

\section{Conflict of Interest}

The authors report no conflict of interest.

\section{References}

1. Nurwahida, Hardianto A, Rizki KA. Multiple sialolithiasis of submandibular gland: a case report. J Dentomaxillofac Sci 2017;2: 129-132.

2. Sapp JP. Contemporary oral and maxillofacial pathology. 2nd ed. St. Louis, Missouri: Mosby; 2004.

3. Rai M, Burman R. Giant submandibular sialolith of remarkable size in the comma area of Wharton's duct: a case report. J Oral Maxillofac Surg 2009;67: 1329-1332.

4. Lewis KK, Herold J. Familial giant cell lesions of the mandible- unusual occurence. J Oral Maxillofac Surg 2013;71: e75-e76

5. Louis DN, Feldman M, Carter AB, et al. Computational pathology: a path ahead. Arch Pathol Lab Med 2016;140: 41-50.

6. O'Connell JE, Kearns GJ. Denosumab: an alternative therapy for the management of giant cell lesions. J Oral Maxillofac Surg 2016;74: 873.

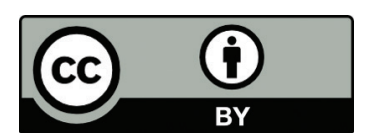

This work is licensed under a Creative Commons Attribution 